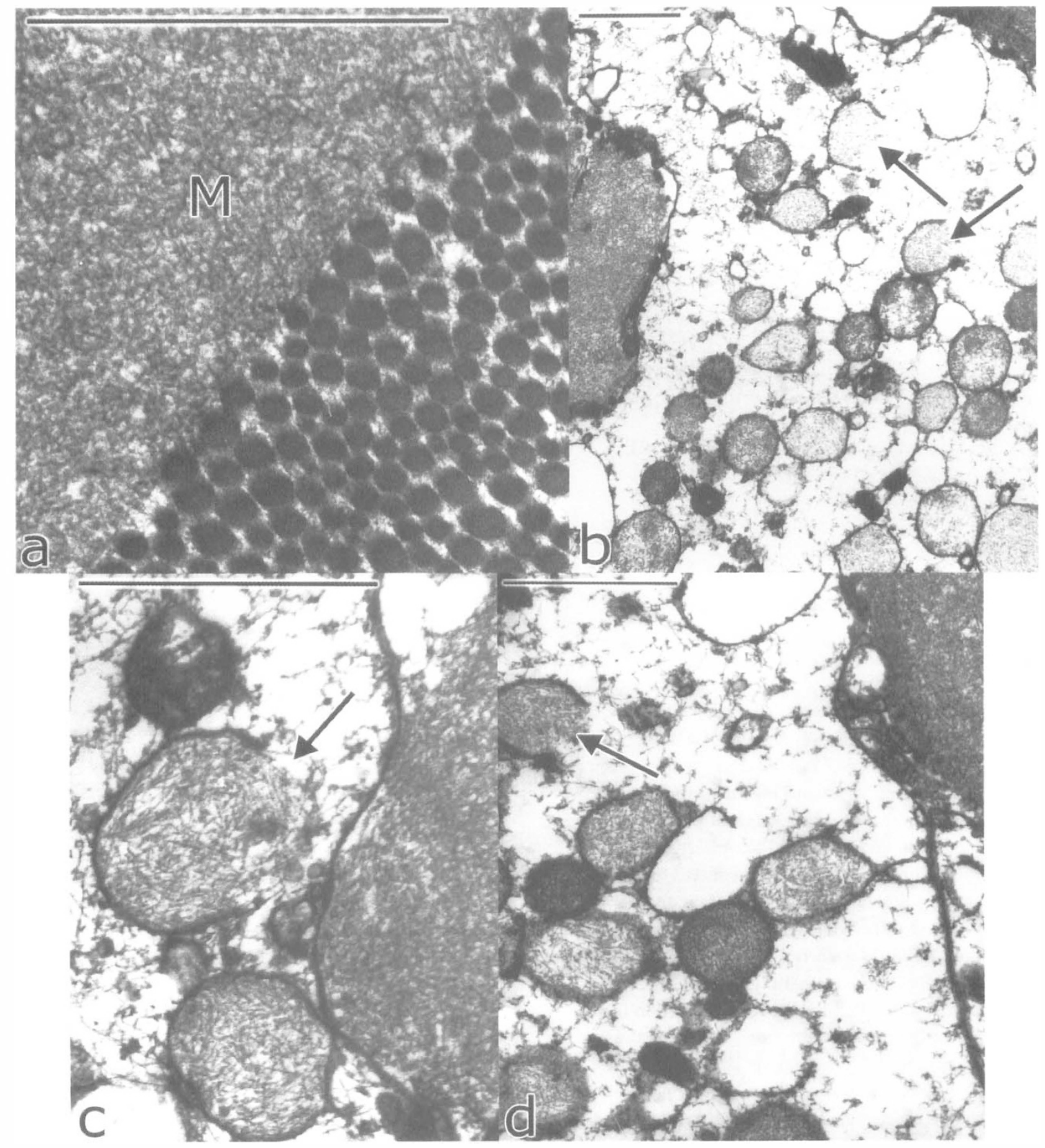

Fig. 3. Electron micrographs (scale bars represents $1 \mu \mathrm{m}$; glutaraldehyde fixation, osmium textroxide staining). (a) The extracellular material ( $M$ ) is electron-dense and composed of randomly arranged short straight or curved non-branching linear filaments around $10 \mathrm{~nm}(100 \AA)$ in diameter, some appearing in a double 'tram-line' configuration. Collagen fibrils 40-80 $\mathrm{nm}(400-800 \AA)$ in diameter are at bottom right. (b)-(d) The cytoplasm of stromal cells contains numerous membrane-bound structures resembling (and consistent with) lysosomes; many of these contain filamentous material of similar structure to the extensive extracellular filamentous material, although occasionally the filaments in the vesicles appear slightly longer $(c, d)$. In occasional cells $(b, c, d)$ the membrane-bound vesicles appear to have ruptured (arrows), releasing the filamentous material into the cytoplasm of the cells. Occasional apparently 'empty' (fluid-filled) vesicles are also noted $(b, d)$, some with ruptured membranes.

Sir,

\section{Retinitis pigmentosa with osteochondrodysplasia in siblings}

It is well known that retinitis pigmentosa (RP) may occur with various diseases such as deafness, obesity and mental retardation. Many systemic diseases, some well defined and others not well understood, have been associated with pigmentary retinopathy. We report on a sister and brother who have RP with osteochondrodysplasia.

\section{Case reports}

Case 1. A 9-year-old girl was referred to a paediatrician for progressive deformation of the thorax. At that time, retinal degeneration was diagnosed. Her parents had noticed her night blindness.

The girl had one younger brother (case 2). There was no consanguineous marriage in the family. None of her relatives showed any abnormality related to body development, structure or ocular history. 


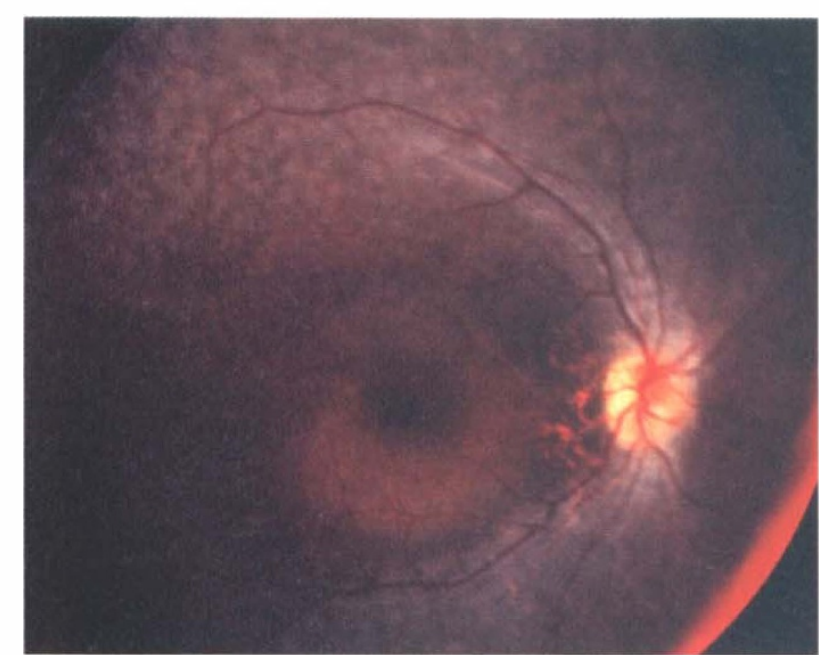

(a)

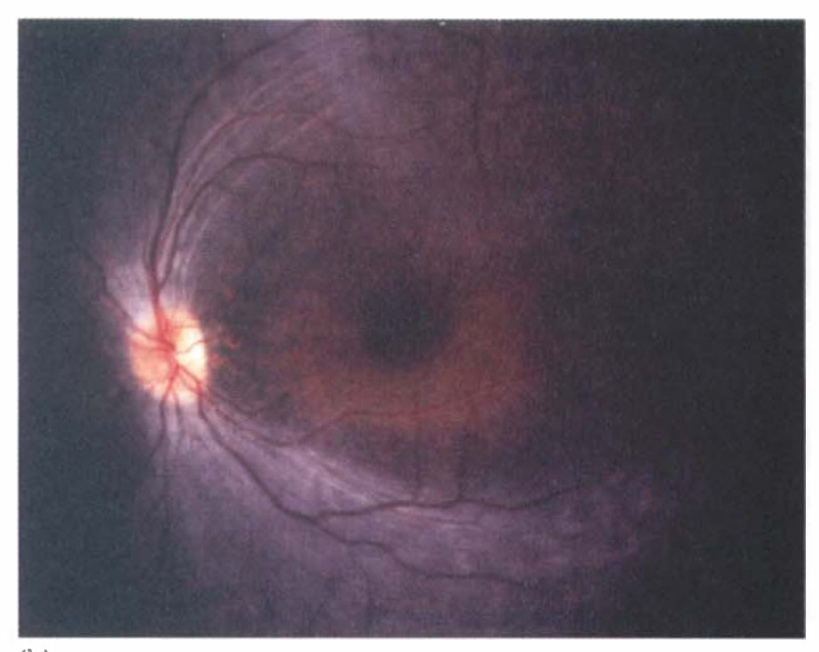

(b)

Fig. 1. Funduscopic photographs. In the right (a) and left (b) eyes of case 1, retinal arteries are slightly narrow and a so-called salt and pepper appearance is seen. Bone spicules were not found.

No perinatal or intranatal abnormality was found. From 4 months of age the girl had low height and weight, and at 9 years was about mean -2 standard deviation (SD) for her age. From 8 months of age thorax deformation was noted, and at age 3 years she had pneumonia because of the reduced respiratory function associated with it.

Her corrected visual acuities were 20/30 in her right eye and 20/50 in her left eye. Results of a slit-lamp examination were normal. Funduscopic examination showed normal optic discs and maculae, slightly narrow retinal arteries, and a so-called salt and pepper appearance in the middle retinal periphery (Fig. 1). No bone spicules were found, and we suspected that she had an early stage of typical RP. The fluorescein angiographic findings showed diffuse background hyperfluorescence and mottling hyperfluorescence in the peripheral retina, based on atrophy of the retinal pigment epithelium. Goldmann visual fields revealed mild reduction of sensitivities in the middle peripheral and central areas. Electroretinograms with dark adaptation showed severe reduction of both a- and b-waves. Goldmann-Weekers dark adaptometry revealed mild elevation of the rod thresholds. No colour vision abnormalities were found by colour vision testing.

Mental and motor development and the balance of face and body development appeared normal. Precordial thoracostenosis was found, and her breastbone protruded forward (Fig. 2a). Roentgenographic findings revealed the protruding breastbone and the concave thorax. Physiological kyphosis of thoracic vertebrae was absent and flat thoracic vertebrae were found. Distal metaphyseal bone trabeculae of the os femoris were obscure. The third os metacarpale of her left hand showed shortening and hypertrophy of the metaphysis.

Routine blood and urine analysis was normal. Based on spirometric findings, the mixture of both obstructive and restrictive lung disease was suspected. Audiometry showed normal hearing.
Case 2. The 3-year-old brother of the proband was referred to a paediatrician for progressive deformation of the thorax. At that time, retinal degeneration was diagnosed.

No perinatal or intranatal abnormality was found. From 6 months of age, low height and weight were noted and at 3 years about mean -2 SD for his age. From age 1 year, thorax deformation appeared and at 2 months and 3 years of age he had bronchitis and pneumonia. His past history was similar to that of case 1 .

His corrected visual acuities were 20/40 in his right eye and 20/50 in his left. Slit-lamp examination revealed normal findings. Funduscopic examination showed almost the same findings as case 1. Electroretinograms showed that the amplitudes of both the a- and b-waves were slightly larger than those of case 1 .

The boy's mental and motor development was normal. Precordial thoracostenosis was found, and the breastbone protruded forward (Fig. 2b).

Roentogenographic findings were almost the same as those of case 1 .

Routine blood and urine analyses and results of audiometry were normal.

\section{Comment}

Various kinds of osteochondrodysplasia are associated with RP, including Jeune syndrome, ${ }^{1,2}$ Saldino-Mainzer syndrome, metaphyseal chondrodysplasia, ${ }^{3}$ Cockayne syndrome, Laurence-Moon-Biedl syndrome, mucosulfatidosis and homocystinuria. Only Jeune syndrome shows deformation of the thorax. Jeune syndrome, ${ }^{1,2,4,5}$ also known as asphyxiating thoracic dysplasia or thoracic-pelvic-phalangeal dysplasia, is an autosomal recessive disorder with thoracic dysplasia (broad, short and horizontal ribs), short-limbed short stature, cone-shaped epiphysis, short phalanges, metaphyseal ossification defects, polydactyly and pelvic abnormalities. ${ }^{1,5}$ Cystic lesions occur in the kidney, liver and pancreas of affected patients. ${ }^{5}$ This syndrome may often lead to death from respiratory insufficiency in 


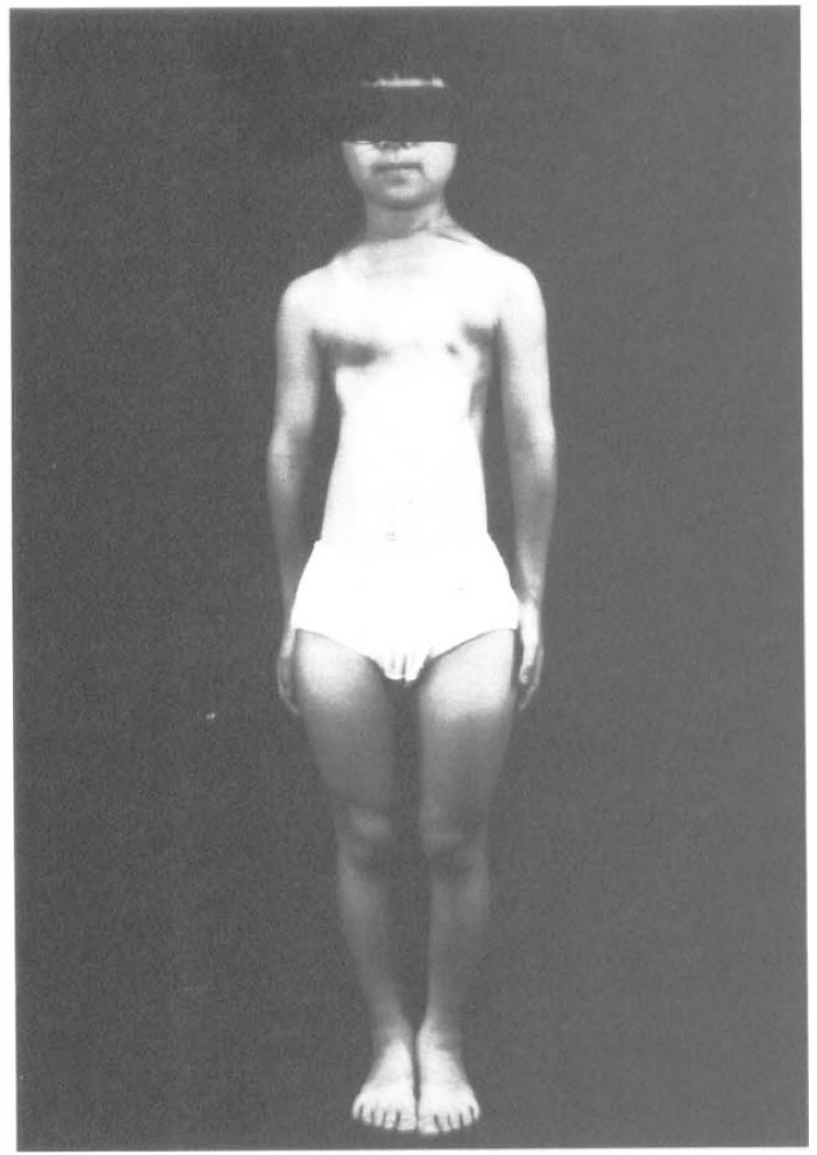

(a)

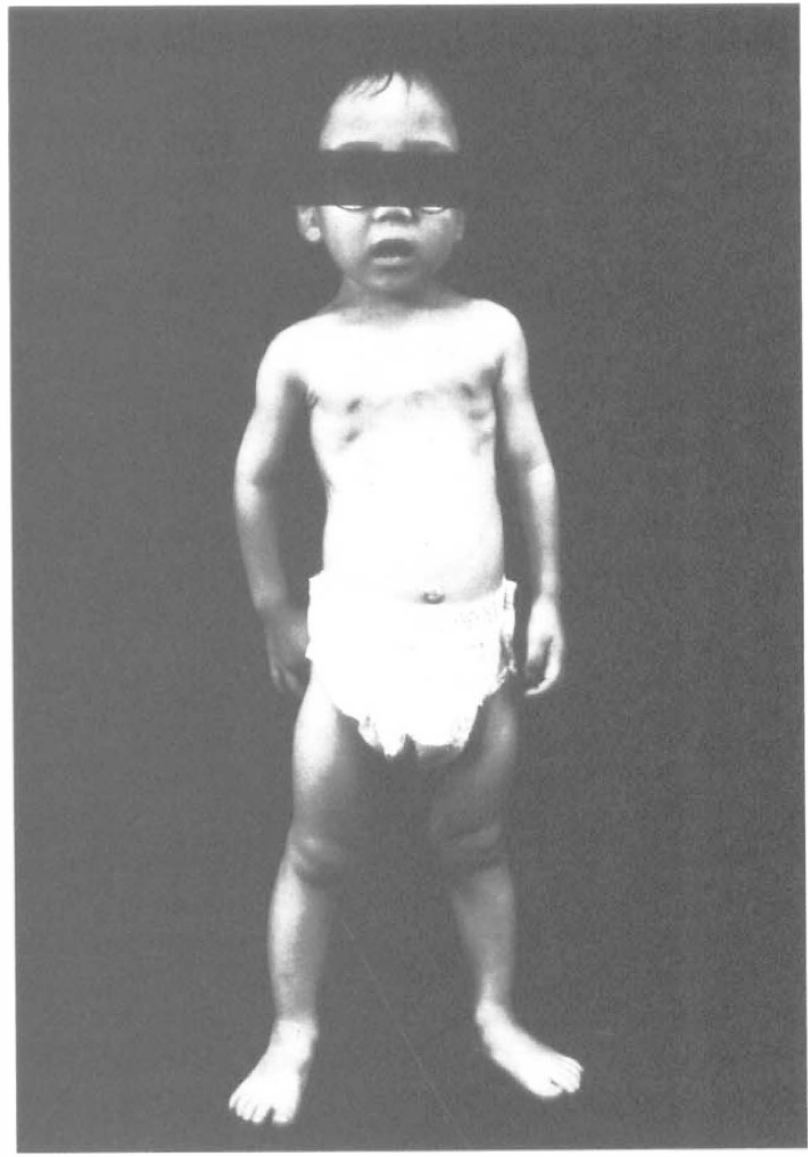

(b)

Fig. 2. Clinical photographs of cases $1(a)$ and $2(b)$. The balance of face and body development is normal, but precordial thoracostenosis is observed, and the breastbone protrudes forward.

infancy, and the renal disease can result in progressive renal failure, necessitating dialysis or kidney transplant. ${ }^{1}$ In our cases, renal function was normal and respiratory insufficiency was not fatal. A mild form of Jeune syndrome, ${ }^{4}$ with only skeletal anomalies and initial renal impairment, has been reported. In our cases, however, bone changes were different from those described in Jeune syndrome. Our cases could not be diagnosed with Jeune syndrome based on the skeletal changes, and are different from reported clinical entities. Further examination and gene analysis are needed.

\section{References}

1. Wilson DJ, Weleber RG, Beals RK. Retinal dystrophy in Jeune's syndrome. Arch Ophthalmol 1987;105:651-7.

2. Phillips CI, Stokoe NL, Bartholomew RS. Asphyxiating thoracic dystrophy (Jeune's disease) with retinal aplasia: a sibship of two. J Pediatr Ophthalmol Strabismus 1979;16:279-83.

3. Phillips CI, Wynne-Davies R, Stokoe NL, Newton M. Retinitis pigmentosa, metaphyseal chondrodysplasia, and brachydactyly: an affected brother and sister. J Med Genet 1981;18:46-9.

4. Giorgi PL, Gabrielli O, Bonifazi V, et al. Mild form of Jeune syndrome in two sisters. Am J Med Genet 1990;35:280-2.

5. Takada F, Hiroki K, Ohkawa Y, et al. Asphyxiating thoracic dystrophy: surgical correction and 2-year follow-up in a girl Jpn J Human Genet 1994;39:269-73.

Mariko Taniai ${ }^{1}$

Atsushi Mizota ${ }^{1}$

Kazuyuki Ishikawa ${ }^{1}$
Mitsuya Ikejiri ${ }^{1}$

Emiko Adachi-Usami ${ }^{1}$

Haruo Kuroki ${ }^{2}$

Masashi Yamazaki ${ }^{3}$

${ }^{1}$ Department of Ophthalmology and Visual Science

${ }^{2}$ Department of Orthopaedic Surgery

${ }^{3}$ Department of Pediatrics

Graduate School of Medicine, Chiba University

Chiba, Japan

Mariko Taniai, MD

Department of Ophthalmology and Visual Science

Graduate School of Medicine, Chiba University

1-8-1 Inohana Chuo-ku

Chiba 260-8670, Japan

Tel: +81432227171

Fax: +81432271810

e-mail: taniai@ophthalm.m.chiba-u.ac.jp

Sir,

Ocular perforation associated with local anaesthetic for dacryocystorhinostomy

The use of local anaesthesia in ophthalmology continues to expand to include not only cataract surgery but also vitreoretinal, strabismus and oculoplastic surgery. The risk of ocular perforation with peribulbar anaesthesia for cataract surgery has been recognised for some time. ${ }^{1}$ We report a case of ocular perforation associated with the administration of local anaesthetic for dacryocystorhinostomy. 